\title{
Juegos de rol e historias ramificadas mediadas por tecnologías como herramientas para la comprensión.
}

\author{
Julio H. Bonelli \\ Centro Educativo Comunitario (CEC) de Casavalle / Universidad del Trabajo del \\ Uruguay (UTU) Juan Acosta 4600 - (598) 99626012 - Montevideo - Uruguay \\ julio.bonelli.beretta@gmail.com
}

\begin{abstract}
RPS and branched scenarios in a e-learning and b-learning for greater understanding by students. We analyze alternatives in different stages of technological availability and our experiences in creating editing development interfaces for non-techies.
\end{abstract}

Resumen. Los juegos de rol y las historias ramificadas en el marco de un curso para una mayor comprensión de los estudiantes. Analizamos alternativas en diferentes escenarios de disponibilidad tecnológica y nuestras experiencias en el desarrollo de interfaces de edición para no expertos en tecnologías.

\section{Introducción}

Nos ha interesado el logro de una mayor comprensión por parte de los estudiantes mediante el uso de recursos digitales, accesibles en diferentes contextos de disponibilidad tecnológica. Tratamos el diseño de situaciones de aprendizaje, métodos de aplicación y evaluación. En particular nos dedicamos a explorar la potencialidad de la narrativa interactiva, lo que nos condujo a crear interfaces que permitieran diseñar y aplicar juegos de rol (JDR) e historias ramificadas (HR). Por medio de estas herramientas procuramos facilitar al docente la aplicación de metodologías activas de aprendizaje centradas en el estudiante y en las relaciones en el grupo de aprendizaje.

Orientando en cuestiones tecnológicas a autores de cursos, tanto en e-learning como blearning hemos constatado que por falta de tiempo recurrían con frecuencia a los foros como único recurso de interacción para tratar los temas. Llegamos a la conclusión que se beneficiarían si dispusieran de herramientas online, aplicables a diferentes campos del conocimiento, que les permitieran crear fácilmente situaciones de aprendizaje eficaces a la hora de lograr la comprensión, permitiendo al mismo tiempo el aprendizaje y la evaluación. Esto nos llevó al desarrollo de aplicaciones de servidor que aprovecharan la potencialidad de la narrativa interactiva. Nos propusimos emplear una interfaz web para que los participantes de un curso participen de una situación problema para resolverla interpretando personajes. Deseábamos “ir más allá” del aprendizaje a partir de un caso o un problema y aprovechar las posibilidades de las aplicaciones de servidor accesibles por la web para lograr un mayor involucramiento del estudiante. Elegimos dos alternativas, los JDR y las HR.

Una posibilidad de trascender la simple presentación de casos o problemas es que los estudiantes intervengan en la situación problema propuesta por el profesor interactuando entre sí a través de la interfaz e interpretando la posición del personaje 


\section{CBIE-LACLO 2015}

Anais dos Workshops do IV Congresso Brasileiro de Informática na Educação (CBIE 2015)

que encarnan y no la propia, un JDR. Por ejemplo, una aplicación en un curso de ciencias sería hacer que los personajes sean varios científicos, relevantes para un tema, con posturas antagónicas. Deben tratar de resolver un problema de interés para los estudiantes. Estos deben interpretarlos para resolver la cuestión, para lo cual necesitan informarse, conocer sus posturas, sus aportes, sus límites, comprendiendo el tema.

Otra alternativa es crear un número finito de posibilidades para que los estudiantes tomen decisiones una o varias veces en pasos sucesivos. Se inventan encrucijadas, tomas de decisiones importantes para el tema. El sistema hace que cada decisión produzca consecuencias. El estudiante puede recorrer algunos de los caminos típicos para resolver un problema seleccionados por el profesor. Se determinan resultados para cada camino. En este caso nos encontramos ante una HR.

La intención es que las interfaces tecnológicas existan en función de la aplicación de metodologías activas de aprendizaje que se centran en el estudiante y en las relaciones en el grupo de aprendizaje. Consideramos el valor de la metodología más allá de la tecnología y combinamos algunos pasos que constituyan un método eficaz para lograr y demostrar la comprensión. A partir de los mismos estamos abordando las posibilidades exclusivas de la tecnología que puedan enriquecer este proceso. Estudiamos diseños usables para facilitar el acceso a la misma por parte de cualquier docente y estudiante.

\section{Fundamentos}

Entendemos por comprensión como lo hace el enfoque de la Enseñanza para la Comprensión de Project Zero, Blythe, T., \& Associates (1998) la capacidad de poder realizar una gama de actividades que requieren pensamiento respecto a un tema, como ser explicarlo, encontrar evidencia y ejemplos, generalizarlo, aplicarlo, presentar analogías y representarlo de una manera nueva.

El medio elegido para lograr la comprensión, las HR y los JDR, pueden relacionarse con el aprendizaje a partir de casos y el aprendizaje mediante problemas. Su uso educativo tiene los mismos fundamentos y se apoya en las principales teorías que tienen un consenso mayoritario de la comunidad educativa, como el constructivismo, la teoría socio cultural de Vygotsky y el enfoque sistémico de la cognición distribuida. En efecto, para el Constructivismo las personas construyen activamente nuevos conocimientos a medida que interactúan con su entorno. Vygotsky en particular resalta el papel de la interacción social, p 56. Sostiene que para comprender las acciones de un individuo se hace necesario, comprender las relaciones sociales en que ese individuo se desenvuelve, conocer el contexto social donde el sujeto se apropia del conocimiento. Entiende que éste está determinado por su propia historia personal y por su interacción como sujeto social.

Por su parte la cognición distribuida propone que la cognición y el conocimiento no se limita a un individuo. Se basa en la coordinación entre individuos y objetos. Hutchins (1980) propone que el conocimiento humano y la cognición no están confinados en el individuo, sino también en espacios de memoria distribuidos, en hechos, o en el conocimiento de los objetos de nuestro entorno. Desde estas perspectivas las HR y los JDR agregan posibilidades respecto al aprendizaje a partir de casos y el aprendizaje mediante problemas, ventajas relacionadas con posibilidades de interacción con el sistema y con otras personas a través del sistema. Las HR suman interactividad a través 


\section{CBIE-LACLO 2015}

Anais dos Workshops do IV Congresso Brasileiro de Informática na Educação (CBIE 2015)

de un software permitiendo aprendizaje por ensayo y error. Los JDR hacen posible la interacción entre los participantes.

Para el Conectivismo el aprendizaje es un proceso de creación de redes. El contenido y los recursos de aprendizaje, en lugar de ser considerados como objetos estáticos, deben ser pensados como un flujo dinámico. Siemens (2006) y Downes (2008) nos recuerdan que en momentos actuales existe abundancia de dispositivos y de información y se hace necesario desarrollar capacidades para seleccionar y utilizar la información útil. Creemos que las HR y los JDR permiten desarrollar estas capacidades.

Se integra, además, el factor lúdico al proceso. Por ejemplo, el uso de JDR nos da la oportunidad de proponer un aprendizaje distendido, lúdico, sin que por ello sea menos eficaz o mas superficial, sino todo lo contrario. Autores como Alvarez, Astd \& Smart Force, en su Clasificación de objetos de aprendizaje por su uso pedagógico, incluyen la “Simulación Juego de Roles” en los “Objetos de práctica” , p.10:

"Los juegos de rol, permiten que los estudiantes construyan y prueben sus conocimientos y habilidades, interactuando con simulaciones realísticas de un escenario de negocios. En ellos, los aprendices interactúan con personajes virtuales y consultan una amplia variedad de recursos, para lograr una serie de objetivos”

Linser (2001) analiza las posibilidades de un JDR Simulación en Ciencias Políticas y afirma que al jugar un papel (en todos estos sentidos), los estudiantes comienzan a identificarse con el papel, lo que les da un interés personal en el procedimiento y aumenta su motivación. Agrega que con el fin de desempeñar funciones con eficacia, los estudiantes deben investigar a través de enlaces en el sitio Web de juego de roles que son seleccionados por el creador de simulación.

Osorio, P. Ángel, M. y Franco, A. (2012) refiriéndose al uso de simuladores educativos para el desarrollo de competencias en la formación universitaria incluyen las HR dentro de los géneros tradicionales de simulación educativa, p. 6.

"En las HR los estudiantes toman decisiones en momentos clave a partir de múltiples alternativas, cada una de las cuales tiene consecuencias diferentes que pueden ser apropiadas o no para la situación dada." ... "Las decisiones tendrán un impacto en la evolución de la historia, terminando finalmente en resultados ya sean exitosos o no exitosos, con la posibilidad de devolverse y ensayar otras alternativas.”

Aldrich, (2009) p.4, pone énfasis en determinados campos de aplicación:

"El mayor atractivo de la utilización de las HR se centra en la simplicidad de su uso, el desarrollo y el estilo en el contenido las hacen altamente pertinentes para vendedores, representantes de centros telefónicos, recién egresados, cargos de servicio al cliente, y administradores en reciente ejercicio. Cualquier persona en posición de alta rotación debería ser entrenada, si bien no exclusivamente, utilizando HR”

Jones, M. \& Devers, C. (2014) subrayan las posibilidades de adaptación al rendimiento individual de cada estudiante de las HR proponiendo modelos que en base a las respuestas de los estudiantes la simulación se adapta utilizando un árbol de ramificación predeterminado, que luego selecciona la siguiente pregunta o escenario para los estudiantes. 
El uso de JDR e HR no consiste en una mera apuesta a la tecnología. Papert a este respecto ha reflexionado acerca de lo que llamó Tecnocentrismo, la falacia de referir todas las preguntas a la tecnología y sus efectos “per se”. Una interfaz de JDR o una historia ramificada no asegura el aprendizaje. Depende de la aplicación. Por otra parte hemos realizado actividades en las cuales los estudiantes proyectan HR en papel, sin computadora. Los métodos empleados toman en cuenta el Construccionismo de Papert (1990) según el cual los sujetos aprenden mejor cuando construyan objetos que les interesen y éstos ofrecen la posibilidad de hacer más concretos los conceptos abstractos o teóricos y más fácilmente comprensibles.

\section{Experiencias en el desarrollo de interfaces}

Nuestro interés por el logro de una mayor comprensión por parte de los estudiantes mediante el uso de recursos digitales surge entre 1998 y 2005 en el período en que participamos en la Universidad Católica del Uruguay (UCU) en el desarrollo de planes experimentales de Educación a Distancia con Learning Space, AVA y Moodle. Posteriormente, entre 2006 y 2010 integrando el Equipo TIC de UCU realizamos el diseño instruccional de cursos sobre la plataforma Blackboard de la Universidad Católica Andrés Bello (UCAB) de Caracas, Venezuela acompañando a otros profesores de México, Colombia, Venezuela y Uruguay que preparaban materiales y debían crear actividades en el marco de una asociación de universidades latinoamericanas, Ausjal. En aquel tiempo en el contexto, en el que trabajamos, la tendencia inicial de la mayoría de los docentes universitarios era recurrir al texto escrito y excepcionalmente al video que los mostraba hablando frente a la cámara. En algunos casos proponían temas mediante fragmentos de películas cinematográficas. La interacción solía darse mediante foros luego de leer o ver el material. Con frecuencia se trataba de docentes que convertían un curso presencial en un curso online con gran formación en sus disciplina.

\subsection{Desarrollos realizados con las herramientas disponibles}

En 2006 no existía la diversidad de servicios online que podemos encontrar actualmente. Nos propusimos crear objetos con narrativa interactiva en formato SWF, el formato más difundido en multimedia interactivo para Internet. Comenzamos a producir estas películas de Flash pero la inversión en tiempo de desarrollo era elevada. Como alternativa utilizamos newsgames y objetos de aprendizaje disponibles en Internet. Llegamos a la conclusión que era necesario producir material propio para crear situaciones de aprendizaje. En los diálogos con los profesores detectamos la necesidad de desarrollar aplicaciones de servidor equivalentes a los servicios conocidos de la Web 2.0, para los fines propuestos, sin requerir conocimientos informáticos ni comunicacionales por parte de los profesores autores.

\subsection{El Juego de Rol}

En 2006 desarrollamos un prototipo de JDR online, El Juego de Rol. Se trató de un sistema beta para uso docente. Lo presentamos con la Prof. Ana María Vacca en el III Simposio Pluridisciplinar sobre Objetos y Diseños de Aprendizaje Apoyados en la Tecnología (od@06) en Oviedo, España, 2006. Nuestro objetivo era crear una aplicación web que permita crear juegos de rol, inscribir usuarios, jugar en línea y evaluar la experiencia. El Juego de Rol hace posible "jugar" con diversos objetivos. Para los jugadores es una herramienta de aprendizaje de fácil uso, que permite 
interacción con el sistema e interacción entre los participantes. El Juego de Rol también es una aplicación para crear diferentes juegos de rol. Admite la autoría para hacerlo adaptable a los objetivos educativos específicos de quien quiera crear su propio juego de rol. La elaboración de la interfaz de usuario buscaba la coherencia con la didáctica que sustentaba el proyecto. En aquel momento diseñamos una interfaz que contemplara los siguientes elementos: Situación-problema, Personajes, Escenarios, Recursos, Mensajería y Eventos.

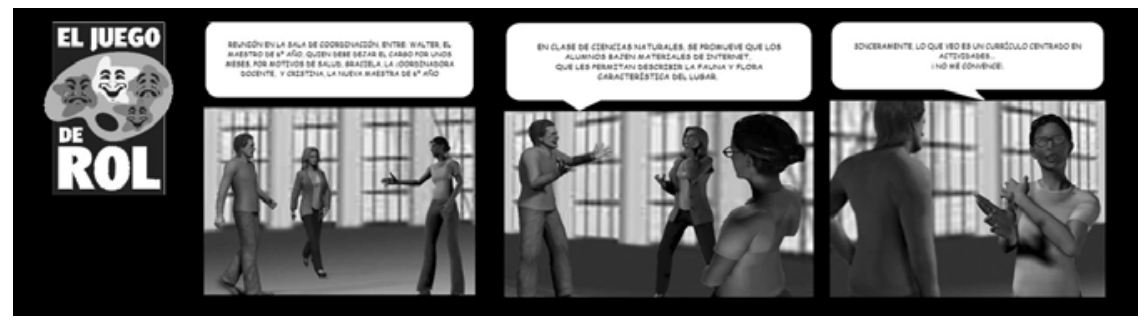

Figura 1. Aplicación de El Juego de Rol para un caso de formación docente, con formato comic y avatares 3D para representar a los personajes. http://eljuegoderol.com/

Como reflexión acerca de los resultados de este proyecto llegamos a la conclusión que era necesario simplificar el proceso de edición. Los docentes con los que trabajábamos en aquel momento preferían algo más sencillo y comenzamos a pensar en la construcción de casos mediante texto.

\subsection{El Samdhastory: Historias colaborativas y el desarrollo colaborativo de guiones}

En 2008 creamos una variación del sistema anterior para permitir a estudiantes de Cine y de Ciencias de la Comunicación crear guiones e modo texto en forma colaborativa. El Samdhastory, fue presentado en Cineteca de la Universidad Católica de Valparaíso, Chile. De utilidad para la creación grupal de guiones, en equipos que no pudieran reunirse en un mismo lugar o conectarse en forma sincrónica, para libretar actuaciones, programas televisivos, películas, spots publicitarios e historias en general. Presentaba la ventaja (al menos teórica) de permitir que personas diferentes pensaran lo que dice y hace cada personaje. Se proponía una autoría grupal desde psicologías múltiples, “como en la realidad” en vez de tener un solo autor pensando lo que hace cada personaje. Luego fue utilizado en centros educativos de Uruguay como JDR en modo texto con otros fines, crear historias en forma colaborativa mediante la inerpretación en línea de personajes en escena.

Si bien el Samdhastory fue una ramificación en el desarrollo inicial de El Juego de Rol no buscaba lograr la comprensión sino la creación de guiones, aunque también fue utilizado con fines didácticos para la discusión de casos, como un JDR en modo texto. El texto era preferido por algunos docentes pero resultaba poco atractivo para los estudiantes, lo que nos llevó a plantearnos el próximo paso, RPStory, una variación de la interfaz para crear juegos de rol, ahora en modo de guión gráfico o story-board, una secuencia de gráficos que muestran lo que hacen los personajes, acompañados de textos que incluyen diálogos y aclaraciones.

\subsection{RPStory: Historias - juego de rol en story-board interactivo}

En 2012 creamos RPStory que permite la construcción de historias-juego de rol. Lo presentamos con la Prof. Ana María Vacca en el Congreso Iberoamericano de las 
Lenguas en la Educación y en la Cultura, OEI, Salamanca, España, 2012. El prototipo para el Congreso se orientaba a la escritura de diálogos pero la herramienta es útil para diferentes campos del conocimiento. En RPStory los profesores pueden crear una situación problema representada por un story-board. La historia inicia, se plantea una situación y los estudiantes deben crear el desenlace interpretando los personajes. Cada participante, deberá argumentar, justificar sus opiniones y comprometer una posición con respecto a la situación conflictiva planteada interpretando el punto de vista del personaje y no el propio. Debe conocer el tema. Como elemento adicional, está la motivación por la simulación lúdica. El final no está determinado en un número finito de opciones como en una historia ramificada, lo crean los participantes libremente.

Desde el punto de vista del aprendizaje se incorpora una novedad. Se agrega un forochat contextual para el desarrollo metacognitivo. En el los estudiantes pueden comunicarse en forma paralela, no como personajes sino como ellos mismos, para resolver que pueden hacer los personajes, para decidir cual reacción se ajustaría mejor al personaje que interpretan. También interviene el profesor o un moderador. Los resultados variaron dependiendo de la actitud del moderador. La tendencia de los estudiantes fue prescindir de este foro-chat metacognitivo cuando el moderador no provocaba su uso adecuado. En estos casos se percibía una mayor superficialidad en los resultados y un alejamiento de los participantes respecto al personaje que interpretaban.

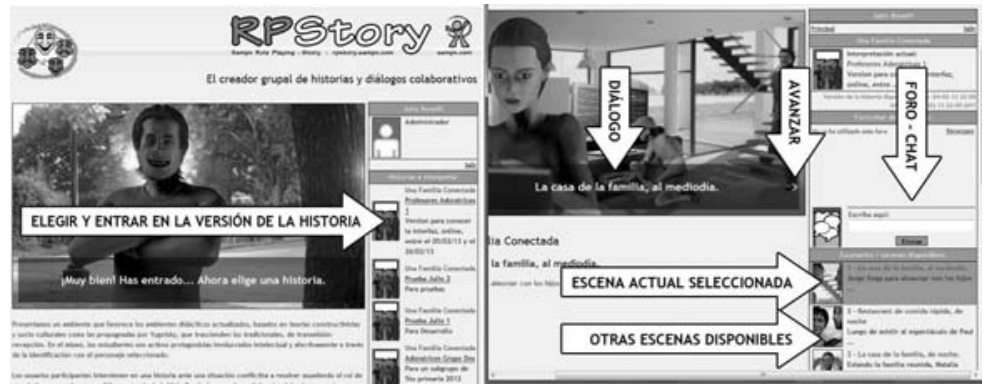

Figura 2. Interfaz del RPStory. Historia Una Familia Conectada. http://rpstory.sampx.com/

RPStory permite la comprensión y la profundización personalizada. El aprendizaje mediante el RPStory no es automático, depende de la participación activa de un moderador consciente de los objetivos de la historia y coherente con la metodología.

\subsection{Interfaz de creación de HR.}

Si bien en los desarrollos anteriores intentamos crear mayores posibilidades de interacción entre los participantes como factor de aprendizaje, con frecuencia los docentes buscaban producir materiales que no les insumieran demasiado tiempo de fabricación, de tutoría y de evaluación. Ideamos una interfaz de creación de HR, sin por ello descartar la alternativa de los juegos de rol.

Actualmente trabajamos en un sistema beta que permite crear HR e investigamos en torno a los métodos de aplicación. Encontramos que en la producción de HR se obtienen buenos resultados si se aplican técnicas de la narrativa audiovisual. Por ejemplo, mencionaremos la estructura dramática, algo que ya aplicamos en RPStory. En las películas se suele dividir el tiempo en tres tercios que se destinan a la presentación, el conflicto y el desenlace. En esta estructura se presentan dos nudos dividiendo el tiempo en tercios. Se trata de momentos de decisiones o acontecimientos importantes para el relato. En la mitad del primer tercio de la mayoría de las películas se trata de presentar 
el tema y todos los personajes importantes. En la primer sexta parte se sabe quién es el protagonista, que quiere y qué se lo impide, un antagonista o un problema. Solamente en el último sexto del tiempo total se puede saber como termina la historia, cual es el final de la película o como ocurrirá, de lo contrario se pierde el interés. Es el momento de las "vueltas de tuerca" de las películas de suspenso. Esta tensión está presente en las películas de ficción y no suele estar presente en numerosos documentales y materiales audiovisuales educativos.

Descubrimos que los participantes de los cursos están habituados a ver películas con esta estructura y suelen desechar documentales y otros materiales que no la toman en cuenta. Puede pensarse que esta preocupación está reservada a los espectáculos, sin embargo quienes desarrollaron la ciencia, el arte, la tecnología, la filosofía han tenido peripecias comparables a las de una película. Intentamos que las situaciones generen este estado de ánimo en los estudiantes para provocar una interacción que genere un aprendizaje. Procuramos que las decisiones que dan lugar a las ramificaciones de la historia tengan lugar en los nudos de la historia 1/3 y 2/3 del tiempo total. Si hay más bifurcaciones procuramos que sean en la presentación y el desenlace (1/6 y 5/6).

\section{Alternativas ante diferentes escenarios de disponibilidad tecnológica}

Las interfaces mencionadas presuponen que los estudiantes y los profesores (autores y tutores) de un curso dispongan de equipos conectados a Internet. Este requerimiento no ha sido un obstáculo insalvable por la generalización de la conectividad y la difusión de diferentes dispositivos. La tecnología en línea se ha vuelto barata y ubicua. Existe una creciente disponibilidad de PC, Laptops, Tablets o Smartphones con conexión a Internet en todo el planeta. La existencia de centros educativos con limitaciones en la conectividad nos dio la oportunidad de avanzar en la reflexión acerca de los métodos. Valoramos la creación de planes de JDR e HR como actividad educativa, mas allá de su utilización y el desarrollo de interfaces que faciliten su creación y aplicación.

En la mayoría de los campos en los que aplicamos las herramientas los participantes de los cursos accedían a equipos con conectividad en el centro y/o en sus casas y/o accedían a equipos móviles conectados a Internet. Desde las perspectivas de una educación inclusiva debemos considerar la viabilidad del diseño de JDR e HR en entornos sin conectividad ni equipos informáticos. Posteriormente hemos comprobado que los pasos a seguir con una interfaz tecnológica para crear una situación de aprendizaje pueden ser dados con papel y lápiz en ausencia de los equipos y conectividad. En efecto, en cursos dados con estudiantes en el CEC de Casavalle perteneciente a UTU hemos propuesto en noviembre y diciembre de 2014 actividades en las cuales los estudiantes crearon story-boards con historias de un personaje que los representaba. Posteriormente se convirtieron en diagramas en papel de HR, con personajes decisiones y diferentes consecuencias. Los CEC atienden un público de adolescentes que abandonaron la educación secundaria, en barrios carenciados de Montevideo.

En una primera etapa se crearon historias lineales dibujadas en papel como storyboards. Una por subgrupo. Todos tenían alguna experiencia de videojuego. El tema era libre. Lo proponían los adolescentes. Por lo general se relacionaba con aspectos de su situación social. Un protagonista quería algo y tenía dificultades para conseguirlo o le ocurría algo e intentaba remediarlo. Los contextos condicionaban las posibilidades y 


\section{CBIE-LACLO 2015}

Anais dos Workshops do IV Congresso Brasileiro de Informática na Educação (CBIE 2015)

otros personajes se oponían o ayudaban. Se trabajó integrando disciplinas como Informática, Matemáticas, Lengua y Filosofía. Para presentar y discutir en plenario los story-boards de las historias empleamos un método basado en el concepto, el pensamiento, su creatividad y su fantasía más que en el uso de la computadora.

Ayudados con papelógrafos, marcadores y cartulina en rectángulos, rombos y flechas hicimos crear diagramas de flujo sin el uso de los laptops. Les propusimos a los estudiantes que las historias ya no fueran lineales, que pudieran tener diferentes finales alternativos. Pensar en el momento previo al desenlace y crear momentos de decisión del personaje, representados por rombos en el diagrama. Para cada decisión que toma el personaje obtiene distintas consecuencias. De esta manera se construyeron diagramas de HR. Inicialmente hubo una tendencia a considerar que los resultados ocurren por causas externas y se restaba importancia a la opción que tomaba el personaje. La reflexión abarcó no solo las opciones seleccionadas para el diagrama sino la discusión sobre todas las alternativas descartadas. Se construyeron diagramas de HR en papelógrafos para cada historia lineal.

Comparamos algunas historias con situaciones reales conocidas por ellos e hicimos notar que los modelos en papel aun eran muy simples para representar casos complejos. El paso siguiente fue proponer que la decisión de un personaje tenga consecuencias para otros personajes de otras historias, pensar la interrelación de las situaciones. Unimos historias con esta finalidad. Era necesario considerar todas las combinaciones de decisiones posibles y para cada una discutir y elegir consecuencias que tengan interés para el tema que se está tratando. Una historia ramificada puede ser eficaz pero a veces es demasiado simple para crear algo que se parezca a un videojuego interesante, o para crear un modelo que represente una situación real, útil para el aprendizaje. Este proceso se completó parcialmente y constituye un paso más allá de una historia ramificada en la cual se toman en cuenta las decisiones de un solo personaje. Un solo jugador no controla el resultado. Se alcanzó un nivel de complejidad que superó lo esperado si tomamos en cuenta que trabajamos con adolescentes con necesidades básicas insatisfechas y dificultades para lograr la abstracción. Creemos que la metodología empleada es válida para ser aplicada en otros contextos y niveles.

\section{Conclusiones}

Aplicaciones de JDR e HR significaron una ventaja para el aprendizaje en relación con métodos anteriores que se basaban en una presentación del tema mediante un material seguido de la discusión mediante de foros. Interpretar los personajes de un caso presentado acerca de un tema e intentar resolver una situación sintiéndose parte de la misma, mediante un JDR o una historia ramificada ofrece oportunidades muy valiosas para la comprensión. La tecnología puede contribuir a la inmersión, permitiendo sentirse parte de la situación presentada y facilitando recursos. También el crear un caso, el idear un JDR o una historia ramificada, para que otros intenten resolver una situación, permite una mayor comprensión que hacer una presentación expositiva sobre el tema, aunque no podamos llegar a crear la historia ramificada o el juego de rol. Creemos que merece ser valorada la metodología, mas allá de la tecnología que dispongamos en un momento o en un lugar. Aplicamos los siguientes pasos como método eficaz para lograr y demostrar la comprensión independientemente de la interfaz tecnológica empleada: 
- El diseño de situaciones de aprendizaje por parte de los profesores. Por ejemplo, un JDR o una historia ramificada sobre un caso o un problema.

- Su aplicación por parte de los estudiantes. Por ejemplo, interpretar un personaje en un JDR o una historia ramificada para resolver una situación.

- Puede haber una tercera etapa en la cual los estudiantes diseñan una situación para mostrar otros aspectos del mismo tema. Por ejemplo, proponer otro JDR para tratar el tema o crear nuevas ramas en una historia ramificada.

Otro método aplicado:

- Se propone a grupos de estudiantes que creen un caso o historia lineal sobre un tema de estudio donde un personaje deba tomar una decisión. Deben pensar un desenlace que se relacione con el tema de estudio y sea relevante para el mismo.

- Después se pide que creen diferentes decisiones posibles convirtiendo el caso en una historia ramificada, para que otros conozcan el tema. La deben planificar en diagrama de flujo. Se emplean rectángulos para las secuencias, rombos para las encrucijadas y flechas para relacionarlos. Deben proponer consecuencias distintas para cada decisión, eligiendo las más importantes para tratar el tema.

- Se discute en plenario acerca de los diagramas grupales y su relevancia para el tema tratado. ¿Las consecuencias propuestas son relacionadas con la decisión y con el tema o con factores externos o el azar?. ¿Son las más pertinentes?

- Diseño de diagramas que crucen dos o más historias tomando en cuenta las diferentes consecuencias para cada una de las combinaciones de decisiones. Las decisiones de un personaje de una historia afectan el desenlace de otra historia.

Notemos que en este proceso es fácil una evaluación centrada en la comprensión. Si los estudiantes logran proponer otro JDR para tratar el tema o crear nuevas ramas en una historia ramificada demuestran la comprensión más claramente que mediante un cuestionario o una monografía. Podrían aplicarse otros métodos igualmente válidos.

Es importante tomar en cuenta que los pasos a seguir con una interfaz tecnológica para crear una situación de aprendizaje pueden ser dados con papel y lápiz en ausencia de los equipos y la conectividad. Constituyen una estrategia valiosa en si misma para lograr la comprensión y para demostrarla. Esto no quiere decir que un software no sea importante para aplicarla. La interfaz tecnológica permite un enriquecimiento de la experiencia, una mayor inmersión en la situación, y favorece el logro de los objetivos.

También apreciamos las potencialidades exclusivas de la tecnología. Un sistema puede manejar mejor casos complejos. Estamos trabajando en una sola interfaz que integre juegos de rol, HR y simulaciones incluyendo inteligencia artificial. Estamos pensando en que los docentes puedan programar eventos azarosos y comportamientos de personajes predecibles o que no respondan siempre de la misma manera, sino en función de condiciones que se puedan dar, combinaciones de múltiples variables. A la vez debe ser una interfaz amigable para docentes y estudiantes de cualquier disciplina, sin conocimientos tecnológicos ni altas habilidades lógicas. 


\section{Referencias}

Bonelli, Julio, Pioli, Ana \& Vacca, Ana M.(2006). Los juegos de rol como objetos de aprendizaje. III Simposio Pluridisciplinar sobre Objetos y Diseños de Aprendizaje Apoyados en la Tecnología (od@06). ISBN:978-84-611-5186-8, Oviedo, 2006.

Blythe, T., \& Associates. (1998). “The teaching for understanding guide”. San Francisco, CA: Jossey-Bass, USA

Downes, S. (2008) “The Future of Online Learning: Ten Years On”. http://halfanhour.blogspot.com/2008/11/future-of-online-learning-ten-yearson_16.html (revisado en agosto de 2015)

Hutchins, E. (1995). Cognition in the wild. Cambridge, Mass.: MIT Press, USA.

Jones, M. \& Devers, C. (2014). Virtual Reality: Adaptive and Branching Scenarios. In J. Viteli \& M. Leikomaa (Eds.), Proceedings of EdMedia: World Conference on Educational Media and Technology 2014 (pp. 2446-2449). Association for the Advancement of Computing in Education (AACE).

Linser R, \& Ip A. (2001) The Technology as: "Evaluation of a Role-Play Simulation in Political Science" The Technology Source , January/February 2001. http://ts.mivu.org/default.asp?show=article\&id=1034. (actualmente no disponible).

Osorio, Paola, Ángel, Mary, Franco, Alejandro. (2012) El uso de simuladores educativos para el desarrollo de competencias en la formación universitaria de pregrado. Revista Q, ISSN: 1909-2814

Papert, Seymour: (1990) A Critique of Technocentrism in Thinking About the School of the Future”. Publicado en “MIT Media Lab epistemology and Learning Memo” No2

Red de Instituciones de Formación Profesional Aportes al debate sobre Objetos de Aprendizaje para el desarrollo de competencias laborales. Montevideo: OIT/Cinterfor, 2013. 96 p. Listado de referencias: p. 89 - 94 Glosario: p. 83 ISBN: 978-92-9088-256-5

Salomon, G. (2001). Cogniciones Distribuidas: Consideraciones psicológicas y educativas. Buenos Aires: Amorrortu Editores.

Siemens, G. (2006). Conociendo el conocimiento. Canadá: Editorial Nodos.

Simmons, Rebecca and Stone Wiske, Martha, Project Zero, Harvard "Teaching for Understanding”, http://www.pz.gse.harvard.edu/teaching_for_understanding.php (revisado en julio de 2015)

Vacca, Ana M, \& Bonelli, Julio (2012) Construcción colaborativa de diálogos, en contexto "juego de rol”, a través de la Web2.0. Congreso Iberoamericano de las Lenguas en la Educación y en la Cultura, OEI, Salamanca, 2012.

Vygotsky, Lev S. “El desarrollo de los procesos psíquicos superiores”, Ed. Crítica, Barcelona, 1979.

Vygotsky, Lev S. “Historia del desarrollo de las funciones psíquicas superiores”, Ed. Científico-Técnica, La Habana, 1987. 